\title{
Background Radiation Studies at LHCb Using Geant4
}

\author{
G. G. Daquino, G. Corti, and G. Folger
}

\begin{abstract}
This paper aims to describe the feasibility studies performed to evaluate the background radiation levels in the $\mathrm{LHCb}$ experiment with Geant4. LHCb is one of the experiments that will operate at the LHC (Large Hadron Collider) under construction at CERN. The simulation toolkit Geant4 has been used to model the interactions of particles with the detector. Geant4 is a software toolkit developed and maintained by a world-wide collaboration of physicists and computer scientists. The principal monitored distributions in this study are the dose and the fluence of certain particles in specific locations of the experiment. The dose is defined as the energy delivered in a volume per unit of the volume mass, while the fluence is defined as the number of particles passing through a surface per unit of area. Energy spectra need also to be evaluated to take into account the energy distribution of these particles, since specific problems in the electronics can be caused by particles of certain energies.

For this purpose, we need to tally doses and fluences using Geant4.

The 2D mapping of doses and fluences of charged hadrons, neutrons, gammas, electrons and positrons on planes put in specific critical areas, where the electronics is located, have been obtained.

Geant4 provides different physics models to describe interaction processes allowing customization of the simulation engine to specific problems. Preliminary results using different relevant physics models are presented.
\end{abstract}

Index Terms-Dosimetry, LHCb, Monte Carlo methods.

\section{INTRODUCTION}

$\mathbf{T}$ HE LHC, currently under construction at the European Organization for Nuclear Research in Geneva, will be the most powerful particle accelerator in the world. It is planned to start operation in 2007; two proton beams are accelerated at $7 \mathrm{TeV}$ in an underground tunnel with a circumference of $27 \mathrm{~km}$. LHCb [1], [2] is one of the five experiments located in a collision point of the accelerator. The experiment is dedicated to the precise measurement of $\mathrm{CP}$ violation and other rare phenomena in the $\mathrm{b}$ system. The single arm spectrometer covers a forward region ranging from $12 \mathrm{mrad}$ to $300 \mathrm{mrad}$ in the horizontal axis and to $250 \mathrm{mrad}$ in the vertical axis. The layout of the detector can be seen in Fig. 1 with the interaction point on the left.

Estimating the background radiation level in an experiment is necessary to understand how the radiation field generated by the collision of the primary protons can affect the electronics.

The possible damage can be essentially divided in two main areas:

Manuscript received July 8, 2005; revised July 16, 2006. This work was supported by CERN.

G. G. Daquino is with JRC-IE, Westerduinweg 3, 1755 ZG Petten, The Netherlands (e-mail: giuseppe.daquino@jrc.nl).

G. Corti and G. Folger are with CERN, CH-1211 Geneva, Switzerland (e-mail: gloria.corti@cern.ch; gunter.folger@cern.ch).

Digital Object Identifier 10.1109/TNS.2006.881544

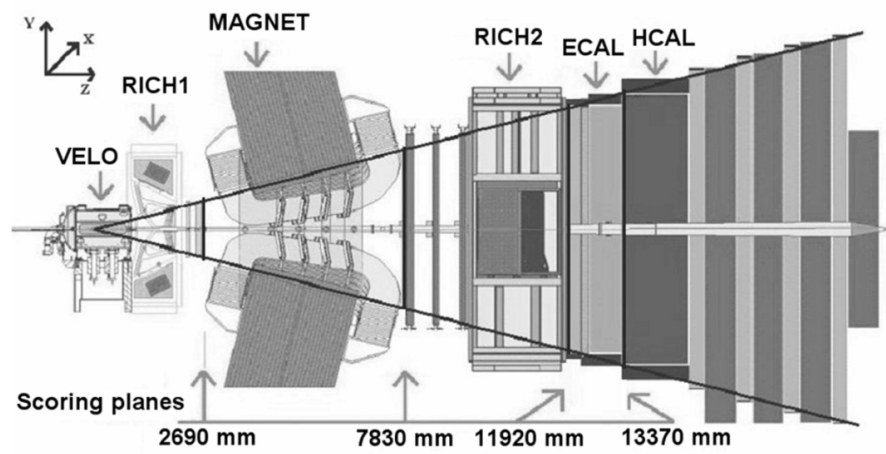

Fig. 1. Vertical section view of the LHCb detector. Some detectors are indicated: the Vertex Locator (VELO), the two Ring Imaging Cherenkov (RICH1 and RICH2) the electromagnetic (ECAL) and hadronic (HCAL) calorimeters The interaction point is on the left while the muon stations are on the right. The solid angle lines represent the main detection area. The four detection planes used for the background radiation studies are also superimposed. The axis directions are indicated.

- gradual effects, taking place during the whole lifetime of the devices exposed to radiation [3];

- local and acute effects, due to the energy deposited by a single particle, which have a certain probability of occurrence that depends on the sensitivity of the devices [4].

Among the gradual effects, the total ionizing dose (TID) is produced by energy deposition of charged particles passing through the device. In the interface regions of semiconductors (insulator layers, contacts, etc.) this leads to accumulation of space charge that distorts the electric field and can produce micro-discharges leading to the degradation of the devices.

Another example of gradual effect is the displacement damage of silicon lattice by Non Ionizing Energy Loss (NIEL) [5]-[7]. This quantity is equivalent to the displacement damage cross section D [5]. Hence the proportionality between the NIEL-value and the resulting damage effects is referred to as the NIEL-scaling hypothesis. $\mathrm{D}$ is normally quantified in $\mathrm{MeV} \cdot \mathrm{mb}$, whereas the NIEL-value is given in $\mathrm{keV} \cdot \mathrm{cm}^{2} / \mathrm{g}$.

An example of local and acute effect is the high energy hadron interaction with the detector device. Such hadrons interact with the material of the electronics producing very high ionization. This strong local charge collection can upset the content of a memory cell and revert individual triggers or switches (Single Event Upset, SEU) or even cause the permanent damage of devices (Single Event Latch-Up, SEL). To evaluate the probability of such events the energy distribution and fluence of the various types of particle has to be convoluted with the specific sensitivities of the devices.

The main aim of this study is to demonstrate first of all the feasibility with Geant4 [8], [9]. This software toolkit is used by Gauss, the LHCb application performing Monte Carlo simulation of the interaction of particles with the detector matter. 

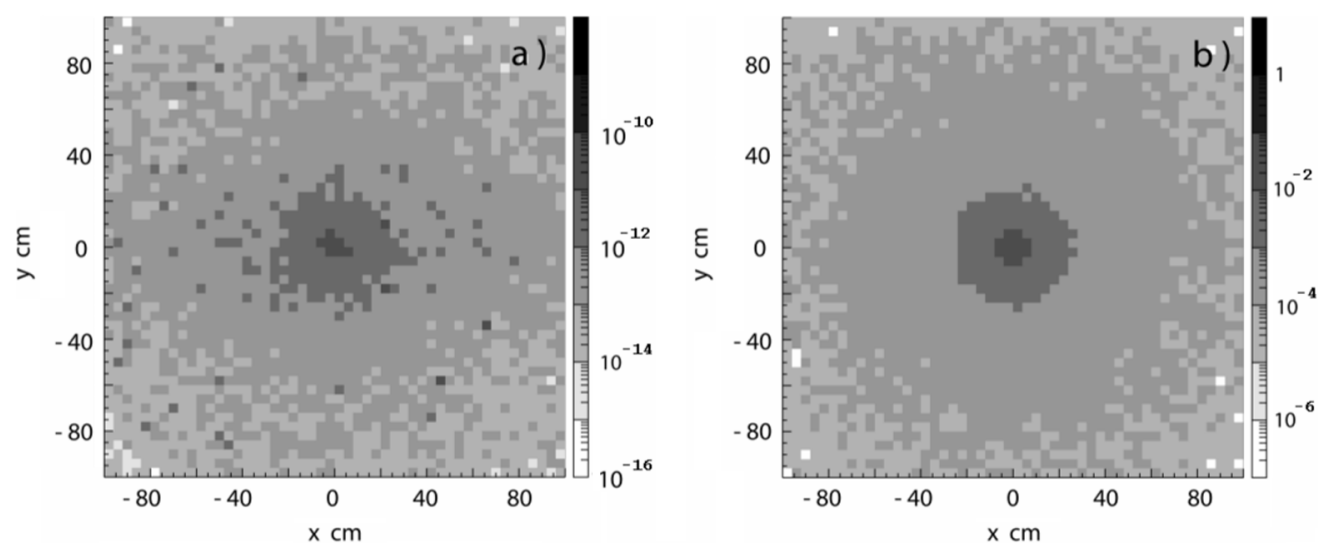

Fig. 2. Total ionizing dose (Gy/collisions) on the scoring plane at $2690 \mathrm{~mm}$ (a); high energy hadron fluence ( $1 / \mathrm{cm}^{2} /$ collisions) distribution at $2690 \mathrm{~mm}$ (b). Both are calculated using LHEP_HP.

\section{MATERIALS AND MethodS}

The main monitoring parameters in background radiation studies are the fluence and the dose delivered by the secondary particles, in the case of LHCb both produced by the collision of two $7 \mathrm{TeV}$ proton beams and by their interactions with the detectors. The dose is defined as the energy delivered in a volume per unit of the volume mass, while the fluence is defined as the number of particles passing through a surface per unit of area.

For this purpose an ad hoc module has been implemented inside Gauss, making use of the Geant4 version 6.2 [8], [9]. The module, called Sensplane, creates detection planes in specific positions, as required by the user configuring the job at run time [10]. The detector planes are defined as $1 \mathrm{~mm}$ thick layers, voxelized in several smaller volumes. Each voxel constitutes an active detector region to record the main quantities (scoring).

The present implementation of Sensplane uses the standard Geant4 navigator for tracking particles. Presently this requires that the scoring planes must be positioned in such a way that there is no overlap with other existing geometry.

\section{A. Fluence and Dose}

The fluence is calculated using the following formula:

$$
\text { Fluence }=\Phi_{p}=\iint_{V} \int_{r} N(\vec{r}, E, t) d r d E \frac{d V}{V}
$$

where:

$-N(\vec{r}, E, t) d r$ is the track length density;

$-\vec{r}, E$, and $t$ indicate the point, energy and time variables respectively;

$-V$ is the voxel volume.

The code estimates this integral by summing $T_{l} / V$ for all the particle tracks in the voxel, time range and energy range. $T_{l}$ is the distance travelled by the particle into the voxel.

The dose is calculated summing the ratios between the energy deposited in the voxel by all the particles of a certain type and the voxel mass for the given material of the detector plane.

The code can be configured at execution time via options provided at start-up. In particular, the user is asked to provide the following input parameters:
- position and dimensions of the detector plane. These specifications are written in an XML file, which is then converted internally by Gauss in the Geant 4 geometry classes. Sensplane takes the geometry information directly from the XML file.

- types of particle which need to be monitored.

The types of particle considered for the background radiation study are limited to those that particularly affect the electronics. The user can choose to collect distributions for one or all of the following:

— protons;

- neutrons;

— charged hadrons (pions, kaons, but not protons);

— electrons and positrons;

— photons;

— high energy $(>20 \mathrm{MeV})$ hadrons.

Based on the user input, the Sensplane provides the following:

$-2 \mathrm{D}$ mappings of the dose and fluence for the user-defined particles.

— energy spectra for the user-defined particles.

\section{B. $1 \mathrm{MeV}$-Neutron Equivalent Fluence}

The displacement damage is the most important cause of damage in the bulk of the semiconductor [7].

In particular, the displacement damage is linked to the fluence of neutrons (especially at low energy) and high energy hadrons.

The evaluation of the displacement damage is performed through the $1 \mathrm{MeV}$-neutron equivalent fluence for silicon that takes into account the different particles contributions as outlined below. The calculation of the $1 \mathrm{MeV}$-neutron equivalent fluence is performed in specific regions of the detector, corresponding to the location of the electronics.

According to the ASTM standard [11], the displacement damage cross section for silicon for $1 \mathrm{MeV}$ neutrons is $D_{n}(1 \mathrm{MeV})=95 \mathrm{MeV} \cdot \mathrm{mb}$, known as the normalising value. On the basis of the NIEL scaling the damage efficiency of any particle with a given kinetic energy $\mathrm{E}$ can then be described by the hardness factor $\gamma$. In general, instead of $D_{\text {particle }}(E)$ 

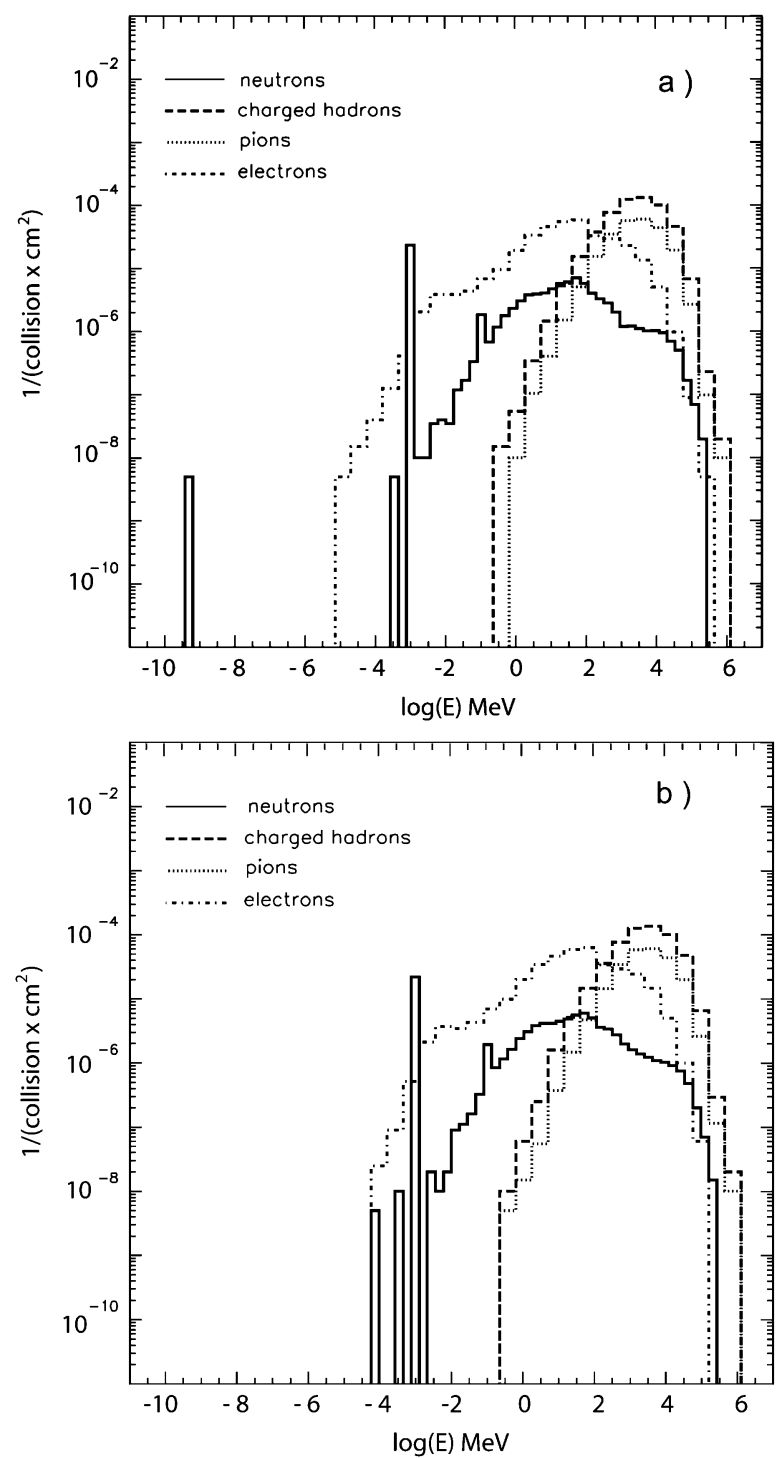

Fig. 3. Particle spectra calculated with LHEP (a) and QGSP (b) at $2690 \mathrm{~mm}$.

the normalized values $D_{\text {particle }}(E) / 95 \mathrm{MeV} \cdot \mathrm{mb}$ are used. The scaling function $D_{\text {particle }}(E)$ is used to calculate the hardness factor. In particular:

$$
\gamma=\frac{\mathrm{EDK}}{\operatorname{EDK}(1 \mathrm{MeV})}
$$

where:

- EDK is the energy spectrum averaged displacement KERMA (Kinetic Energy Released in MAtter) [5]

$$
\mathrm{EDK}=\frac{\int D(E) \cdot \phi(E) d E}{\int \phi(E) d E}
$$

$-\phi(E)$ is the differential flux;

$$
D(E)=\sum_{k} \sigma_{k}(E) \int d E_{R} f_{k}\left(E, E_{R}\right) P\left(E_{R}\right)
$$
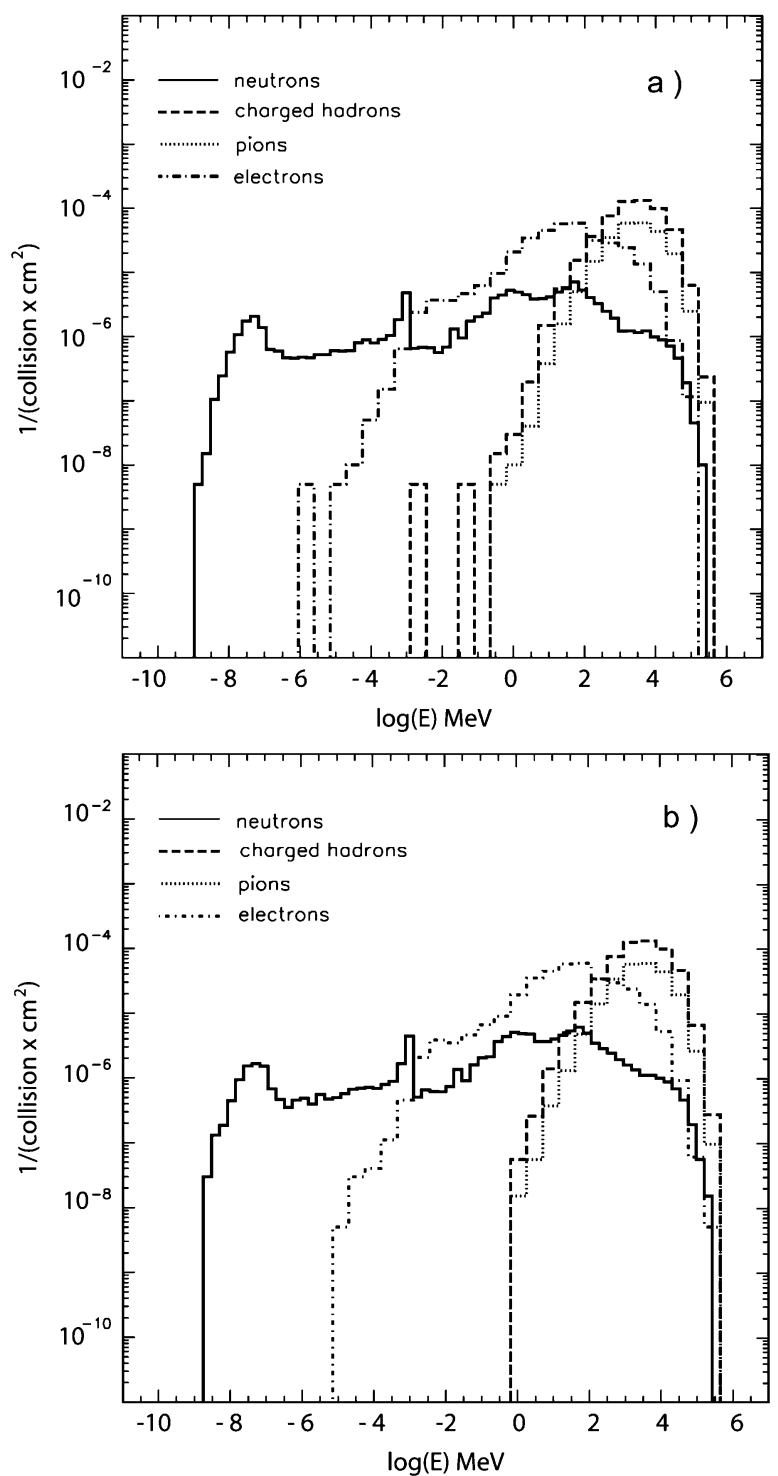

Fig. 4. Particle spectra calculated with LHEP_HP (a) and QGSP_HP (b) at $2690 \mathrm{~mm}$

$-\sigma_{k}$ is the cross section for reaction $\mathrm{k}$;

$-f_{k}\left(E, E_{R}\right)$ is the probability of the incident particle to produce a recoil particle of energy $E_{R}$ in reaction k;

$-P\left(E_{R}\right)$ is the partition function (the part of the recoil energy deposited in displacement).

$-\operatorname{EDK}(1 \mathrm{MeV})=95 \mathrm{MeV} \cdot \mathrm{mb}$.

Equation (2) is used to calculate the hardness factor related to each particle type.

The integration for calculating EDK is done over the whole energy range of the particles.

The hardness factor $\gamma$ is used as a multiplication factor for the fluence distribution $\Phi_{p}$, as defined in (1), related to a certain type of particle, therefore:

$$
\Phi_{\mathrm{eq}}^{1 \mathrm{MeV}}=\gamma \cdot \Phi_{p}
$$

Subsequently, the $1 \mathrm{MeV}$-neutron equivalent fluence for silicon is calculated as sum of the weighted fluence contributions of 


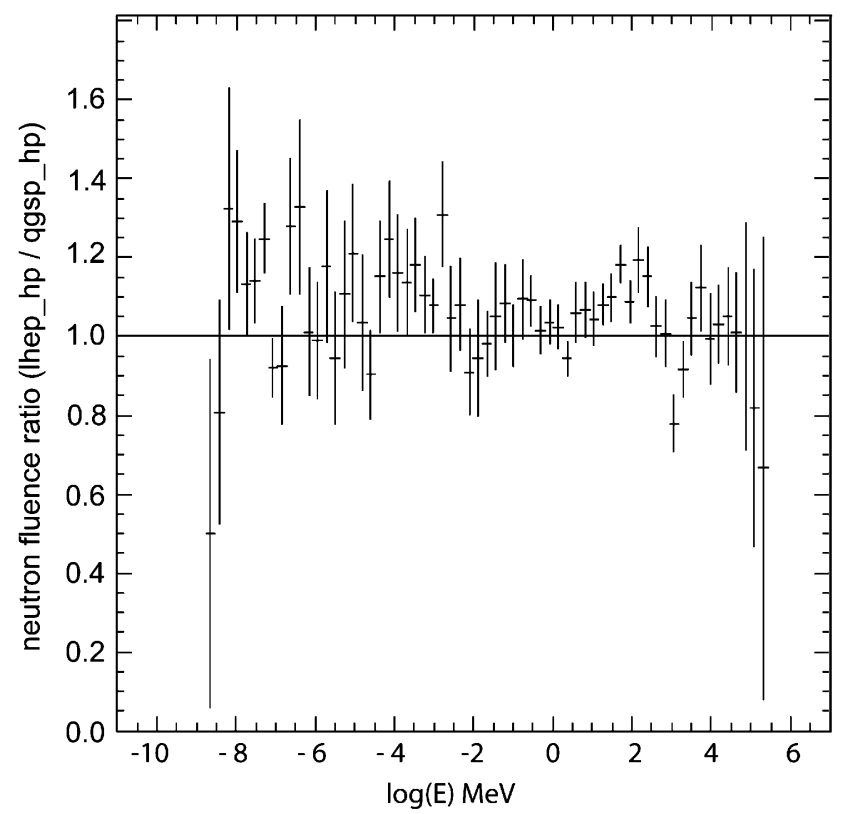

Fig. 5. Ratio of neutron spectra between LHEP_HP and QGSP_HP at $2690 \mathrm{~mm}$.

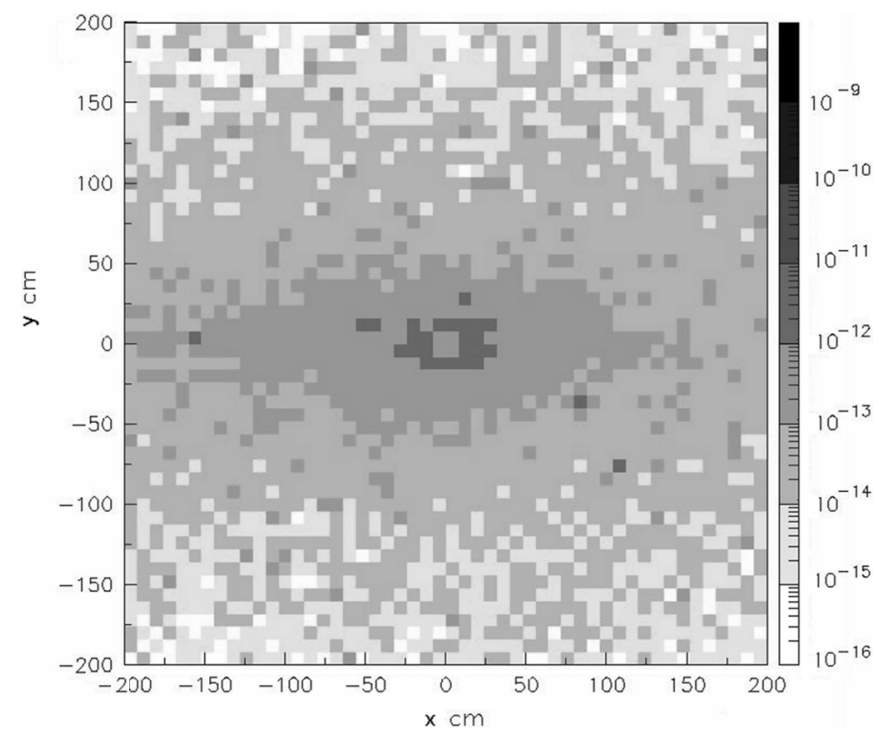

Fig. 6. TID (Gy/collisions) 2D distribution at $7830 \mathrm{~mm}$ (LHEP_HP).

each particle type, obtained with (5). To estimate the $1 \mathrm{Mev}$-neutron equivalent fluence, Sensplane registers the energy spectra of each user-defined particle type.

In the post-processing phase, the energy spectra are convoluted with the damage functions (4), according to (3).

\section{Background Radiation Evaluation at $\mathrm{LHCb}$}

The study reported in this paper refers to the evaluation of all these parameters using the Sensplane module in Gauss in specific positions located inside the LHCb geometry.

Fig. 1 shows the LHCb geometry and the positions of the chosen fake detector planes. In particular the scoring planes are perpendicular to the beam axis and located at different distances from the vertex point:

— the first plane at $2690 \mathrm{~mm}$ (on the left of Fig. 1);
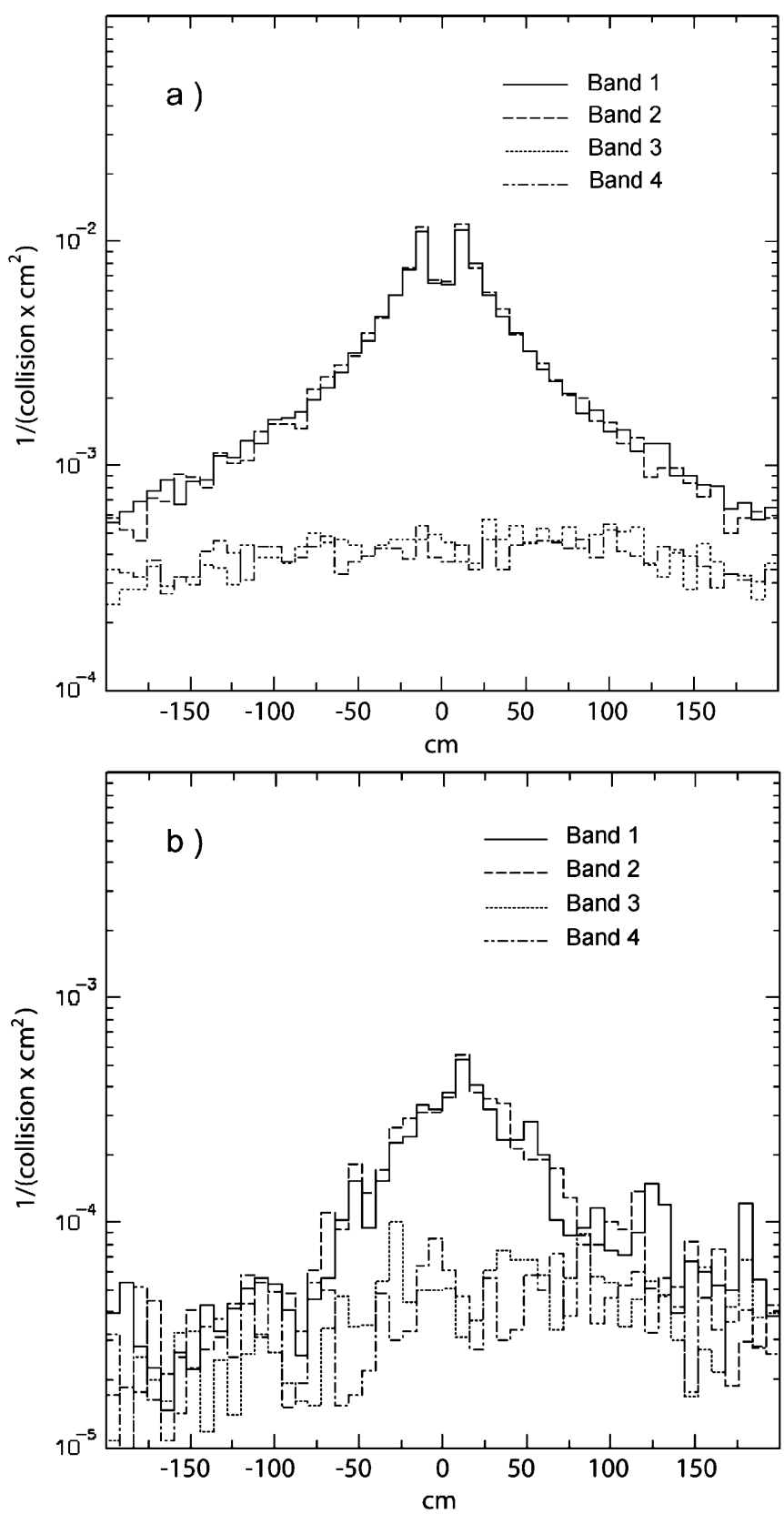

Fig. 7. Total charged hadron fluence (a) and proton fluence (b) around the beam line at $7830 \mathrm{~mm}$, calculated with LHEP_HP. The histograms represent the values integrated on 4 different bands of the y axis positioned around the beam line, located at $\mathrm{y}=0$ : band 1 contains the pixels between $\mathrm{y}=0$ and $\mathrm{y}=-72 \mathrm{~cm}$, band 2 contains the pixels between $\mathrm{y}=0$ and $\mathrm{y}=+72 \mathrm{~cm}$, band 3 contains the pixels between $\mathrm{y}=+72 \mathrm{~cm}$ and $\mathrm{y}=+144 \mathrm{~cm}$, band 4 contains the pixels between $\mathrm{y}=-144 \mathrm{~cm}$ and $\mathrm{y}=-72 \mathrm{~cm}$.

— the second plane at $7830 \mathrm{~mm}$, just after the dipole magnet;

— the third plane at $11920 \mathrm{~mm}$, just before the pre-shower station (PS);

— the fourth plane at $13370 \mathrm{~mm}$, between the electromagnetic (ECAL) and the hadronic (HCAL) calorimeters.

The dimensions of the scoring planes have been chosen in order to match the angular acceptance of the spectrometer. The Sensplane module has been used for the evaluation of the following parameters on the four planes:

— total ionizing dose; 

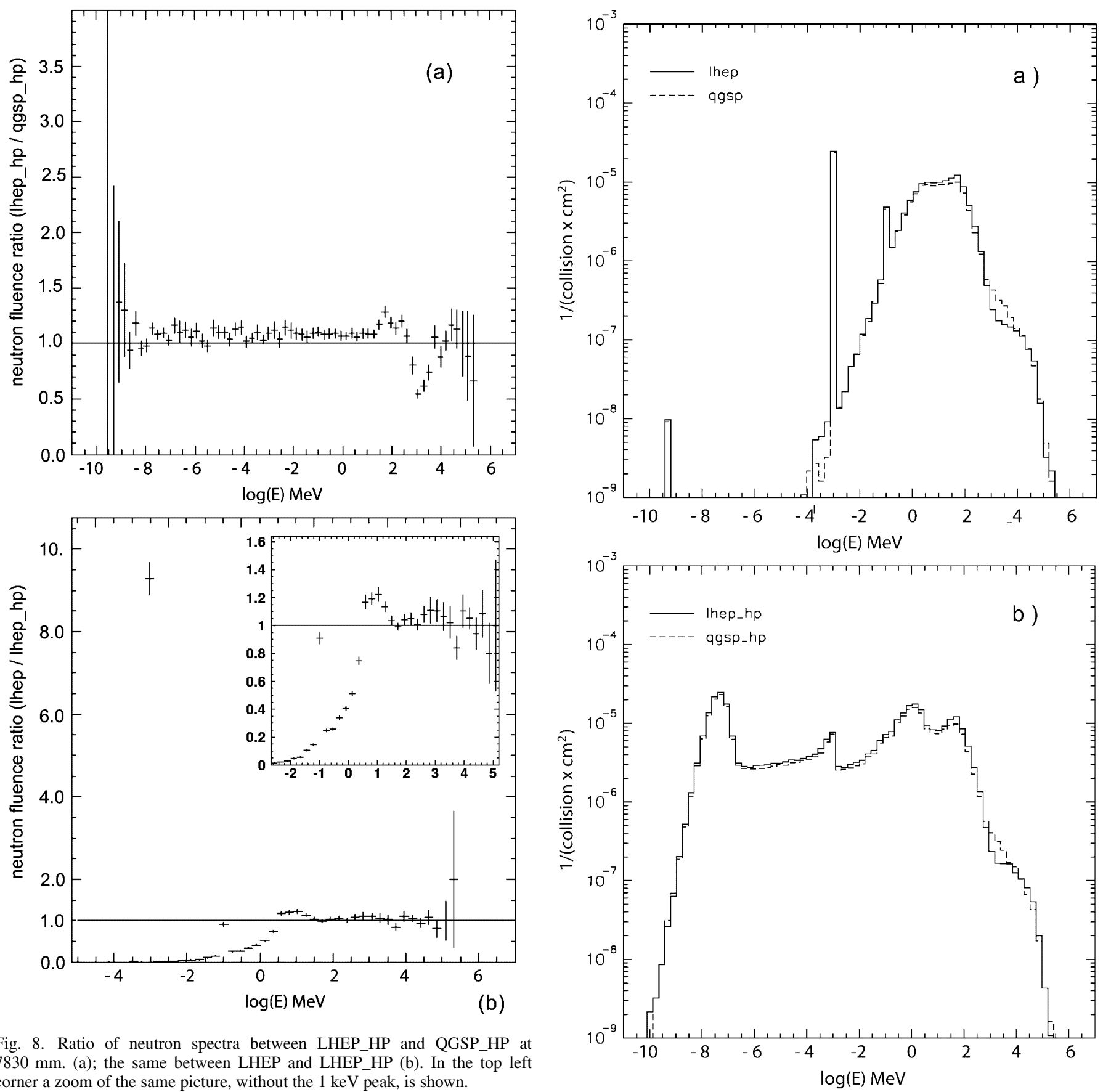

Fig. 8. Ratio of neutron spectra between LHEP_HP and QGSP_HP at $7830 \mathrm{~mm}$. (a); the same between LHEP and LHEP_HP (b). In the top left corner a zoom of the same picture, without the $1 \mathrm{keV}$ peak, is shown.

- proton fluence;

— charged hadron fluence;

- neutron fluence;

- electron/positron fluence;

— high energy ( $>20 \mathrm{MeV}$ ) hadron fluence.

The $1 \mathrm{MeV}$-neutron equivalent fluence for silicon is evaluated in a post-processing stage.

Geant 4 provides a wide variety of models to describe hadronic processes in different energy ranges. In some cases more models cover the same energy range. Physics lists combining several models adequate to various fields of applications are provided in Geant 4 . Six Geant 4 hadronic physics lists have been investigated for the present study [12], [13]:

— QGSP (Quark Gluon String Precompound);

Fig. 9. Neutron spectrum at $11920 \mathrm{~mm}$ calculated with LHEP and QGSP (a); the same obtained with LHEP_HP and QGSP_HP (b).

— LHEP (Low High Energy Parametrized);

— QGSP_HP, similar to QGSP with the additional treatment of the low energy $(<1 \mathrm{keV})$ neutrons. It uses point-wise evaluated cross-section data to model neutron scattering cross-sections and interactions from thermal energies to $\sim 20 \mathrm{MeV}$. This applies to capture, elastic scattering, fission and inelastic scattering.

— LHEP_HP, similar to LHEP with the additional treatment of the low energy $(<1 \mathrm{keV})$ neutrons. The additional features are identical to the ones present in QGSP_HP.

— QGSP_BERT_HP, is similar to QGSP_HP, but it replaces the parametrized model with the Bertini cascade. 


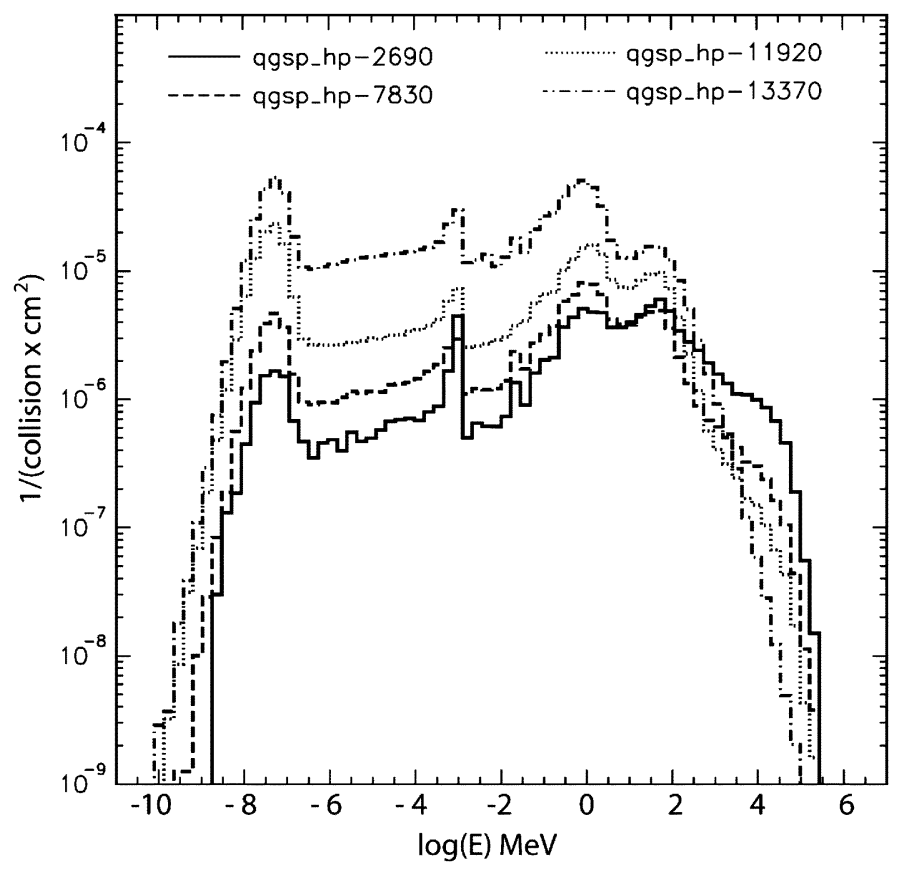

Fig. 10. Neutron spectrum evolution along the different scoring planes, as calculated by QGSP_HP.

—LHEP_BERT_HP, is similar to LHEP_HP, but it replaces part of the parametrized model with the Bertini cascade.

\section{RESULTS}

We present here only the main results obtained using the Sensplane module on the planes indicated in Fig. 1. The plots are shown to give an example of the obtained results. The primary particles statistics corresponds to 5000 proton-proton collisions produced by the Monte Carlo event generator Pythia 6.224 [14]. In the following 2D histograms we show the dose and the fluence per unit of primary event (i.e., proton-proton collision). The 2D histogram shows the results looking at the planes in the $\mathrm{LHCb}$ reference system (indicated in Fig. 1).

The results are focused on investigating the differences among the six outlined physics lists. The same primary events are generated for the six samples by appropriate settings of random numbers for the generator. The primary events contain the expected fraction of elastic, inelastic and diffractive events.

\section{A. Scoring Plane at $2690 \mathrm{~mm}$}

The total ionizing dose for the plane at $2690 \mathrm{~mm}$ shows a centrally peaked distribution, particularly for the LHEP_HP and QGSP_HP models. Fig. 2(a) shows this 2D distribution related to LHEP_HP.

High energy hadrons show a symmetric azimuthal distribution, as shown in Fig. 2(b). This is expected, given the position of this plane within the magnetic field.

Only a few neutrons are present at this position, most of them coming from the primary interaction.

Fig. 3(a) and (b) show the particle spectra calculated with LHEP and QGSP, while Fig. 4(a) and (b) show the same spectra calculated with LHEP_HP and QGSP_HP respectively.

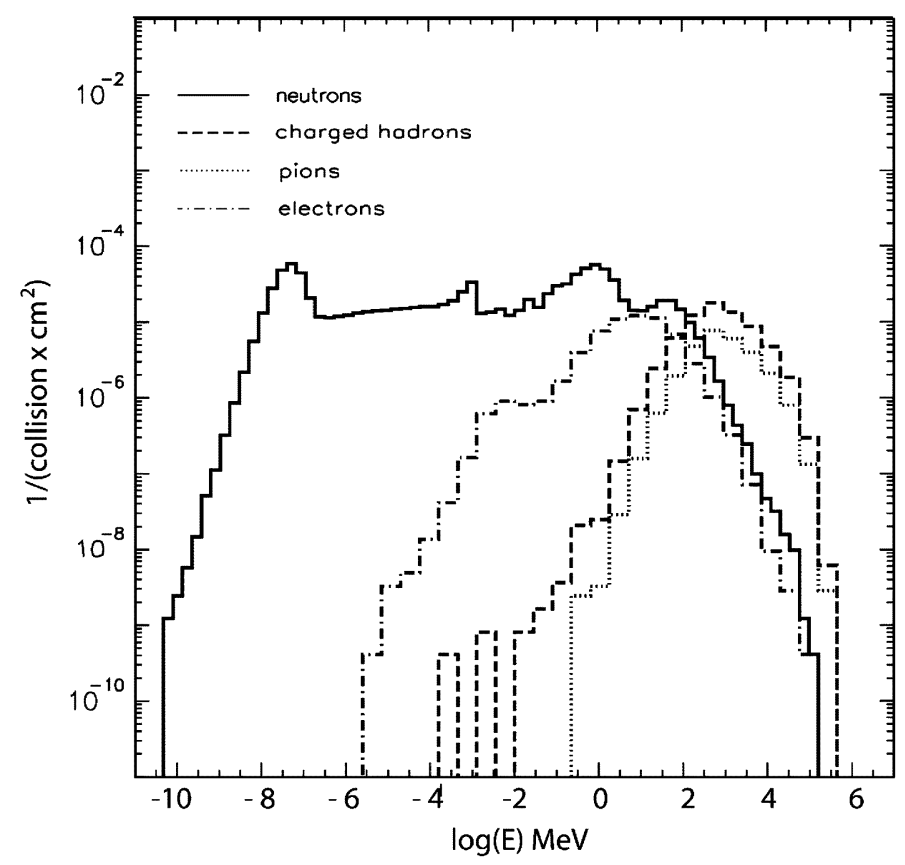

Fig. 11. Particle spectra calculated with LHEP_HP at $13370 \mathrm{~mm}$.

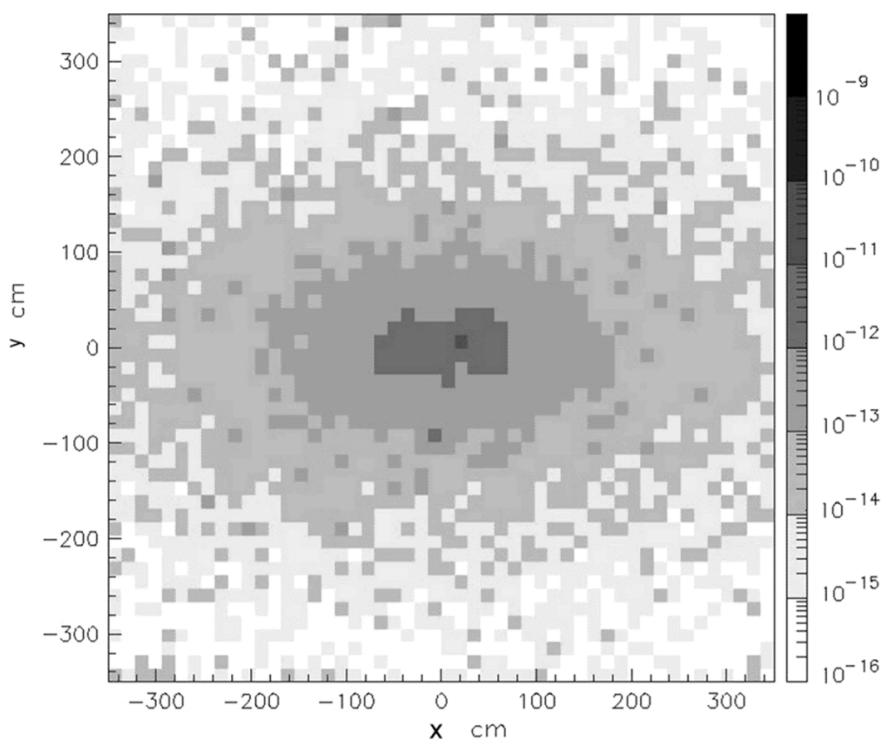

Fig. 12. TID on the scoring plane at $13370 \mathrm{~mm}$ (calculated with QGSP_HP).

It is evident that the simulation of the low energy neutrons requires the HP extension in the physics lists, given the absence of any value in the energy below $10^{-6} \mathrm{MeV}$. The presence of the peak around $10^{-8} \mathrm{MeV}$ is justified by the thermalization process of the neutrons. In fact, the scattering of low energy neutrons in matter slows them down to the thermalization energy $(0.025 \mathrm{eV})$, increasing the neutrons population around $10^{-8} \mathrm{MeV}$. Besides, the peak positioned at $1 \mathrm{keV}$ is connected to the evaporation code of LHEP and is discussed later.

The comparison of the neutron energy spectra of LHEP_HP and QGSP_HP shows that they are similar, see Fig. 5. 

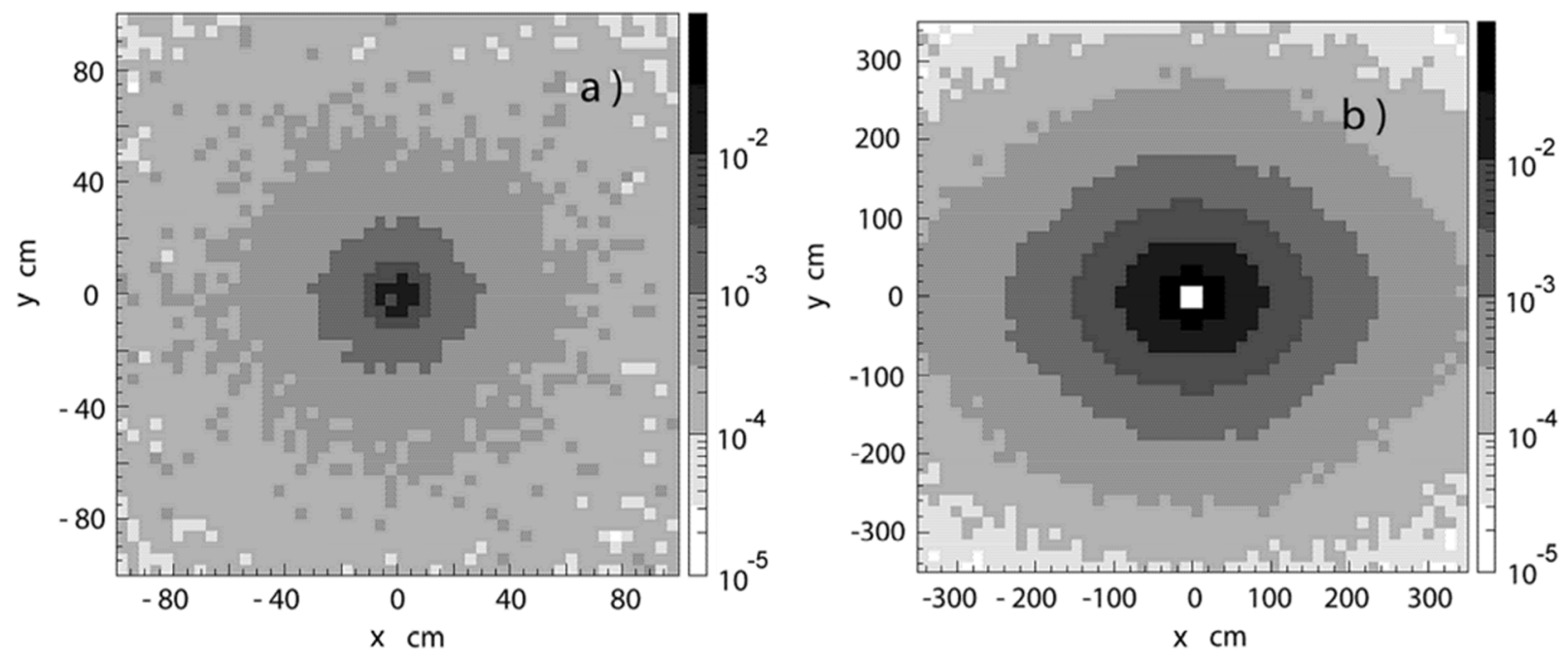

Fig. 13. $1 \mathrm{MeV}$-neutron equivalent fluence 2D distribution at $2690 \mathrm{~mm}$ (a) and $13370 \mathrm{~mm}$ (b) scoring planes.

\section{B. Scoring Plane at $7380 \mathrm{~mm}$}

Charged particle results should be affected by the presence of the magnet. In fact, the total ionizing dose distribution is spread out in the $\mathrm{X}$ axis (the bending direction of the dipole magnet), as is visible in Fig. 6. The results shown have been obtained using LHEP_HP, but no significant differences are present between different physics lists.

The distribution of the total charged hadron fluence along the $\mathrm{X}$ axis is shown in Fig. 7, calculated with LHEP_HP. The effect of the magnet is clearly visible in the proton distribution where an evident asymmetry is present.

A check between different physics lists has been done in order to understand the response of the physics models to the neutrons treatment, especially at low energies $(<20 \mathrm{MeV})$.

For the scoring plane at $7830 \mathrm{~mm}$ the ratio between LHEP_HP and QGSP_HP is shown in Fig. 8(a).

The reason of the visible disagreement present in the region between 1 and $10 \mathrm{GeV}$ is unclear. Two other physics lists (LHEP and QGSP) do not provide an accurate model below $10 \mathrm{MeV}$; hence no comparison with the HP lists is meaningful in the low energy region. Fig. 8(b) illustrates the ratio between LHEP and LHEP_HP.

These comparisons show a general agreement in the energy range specifically covered by the physics lists.

On the other hand the physics lists are not expected to provide identical results.

The isolated point at $\sim 1 \mathrm{keV}$ in Fig. 8(b) is due to a peak in the neutron energy spectra seen with LHEP, QGSP and their HP extension physics lists. It is related to an artefact of the evaporation code of the parameterized model (see Figs. 3 and 4). The peak is less evident in the HP extension lists where additional treatments of low energy neutrons are introduced and replace in most cases the use of the parameterized model. The introduction of the Bertini cascade further reduces the peak until it disappears with the use of the QGSP_BERT_HP physics list as will be discussed later.

\section{Scoring Plane at $11920 \mathrm{~mm}$}

The scoring plane at $11920 \mathrm{~mm}$ has an area of $6100 \times$ $6100 \mathrm{~mm}^{2}$ and is positioned after the RICH2 and before the pre-shower detector. Neutrons are still uniform in azimuth, but the fluence is increased by 4 times with respect to the previous plane, due to the production of secondary particles.

Fig. 9(a) shows the neutron spectrum obtained using LHEP and QGSP, while Fig. 9(b) shows the same spectrum obtained using LHEP_HP and QGSP_HP.

\section{Scoring Plane at $13370 \mathrm{~mm}$}

This scoring plane has an area of $7000 \times 7000 \mathrm{~mm}^{2}$ and is positioned between the ECAL and HCAL of LHCb.

Fig. 10 shows how the neutron spectrum, calculated with QGSP_HP, changes from the $2690 \mathrm{~mm}$ to the $13370 \mathrm{~mm}$ scoring plane.

Particularly evident is the increase of the neutrons population on the scoring plane at $13370 \mathrm{~mm}$ located between the electromagnetic and hadronic calorimeters.

The neutron energy spectrum reaches values of the order of $2 \cdot 10^{-5}$ neutrons $/\left(\mathrm{cm}^{2} \cdot\right.$ collision $)$ in average over the entire energy range with the HP extension.

Fig. 11 represents the particle spectra calculated with LHEP_HP. The charged particles fluence is reduced compared to the previous planes. Consequently, the total ionizing dose is lower and concentrated around the beam line. Fig. 12 shows the TID 2D distribution, calculated with QGSP_HP.

The increase of the neutron production in the last scoring plane is also evident in the $1 \mathrm{MeV}$-neutron equivalent fluence 2D distribution. Fig. 13 shows the increase of the neutron distribution calculated with QGSP_BERT_HP at $2690 \mathrm{~mm}$ (a) and at $13370 \mathrm{~mm}$ (b) far from the $\mathrm{LHCb}$ vertex.

\section{DISCUSSION}

A more detailed analysis of the results of the 2D distributions is obtained monitoring the projections of the vertical bands onto the horizontal axis. A comparison of the six physics lists for the neutron fluence identifies that QGSP_BERT_HP produces 


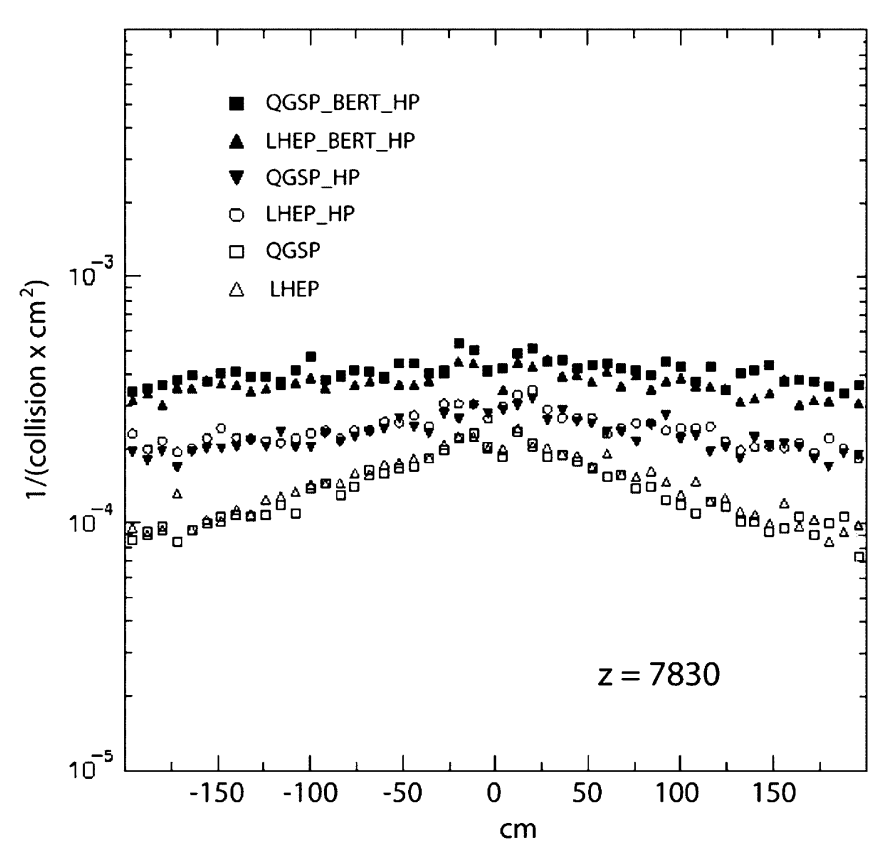

Fig. 14. Comparison among all the six physics lists for the neutron fluence distribution. Band comprised between $\mathrm{Y}=-72 \mathrm{~cm}$ and $\mathrm{Y}=0$.

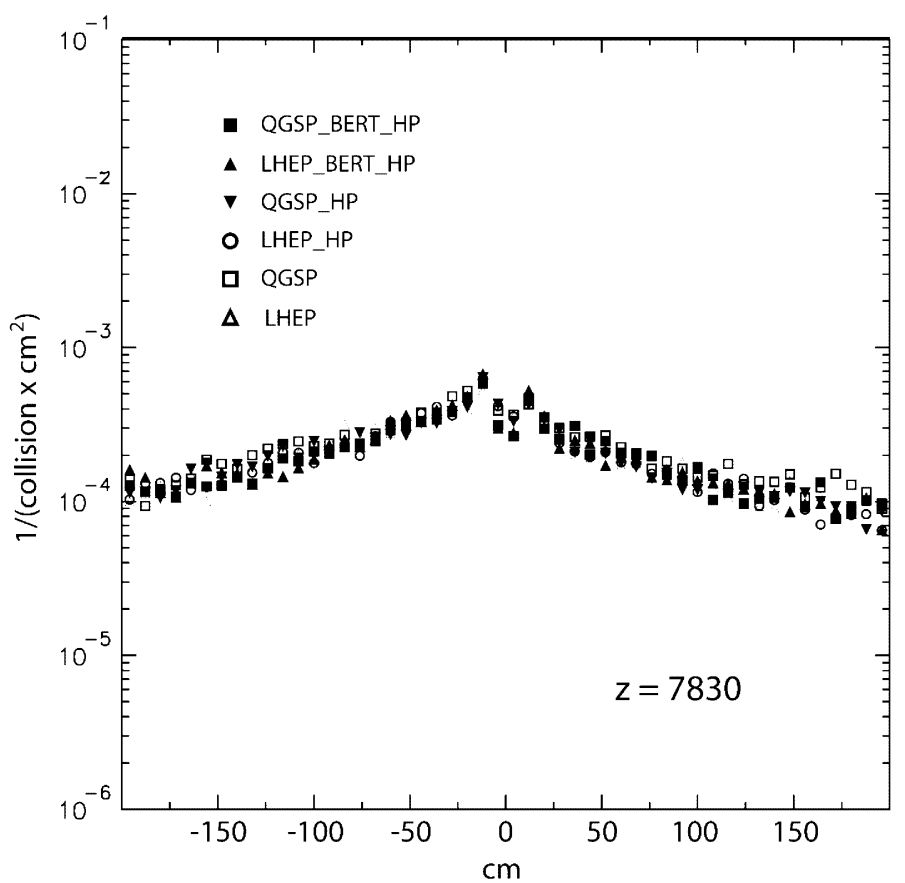

Fig. 15. Comparison among all the six physics lists for the electron fluence distribution. Band comprised between $\mathrm{Y}=-72 \mathrm{~cm}$ and $\mathrm{Y}=0$.

many more neutrons than the other physics lists, as can be seen from Fig. 14 which illustrates the same band (between $\mathrm{Y}=$ $-72 \mathrm{~cm}$ and $\mathrm{Y}=0$ ) for all the physics lists on the $7830 \mathrm{~mm}$ plane.

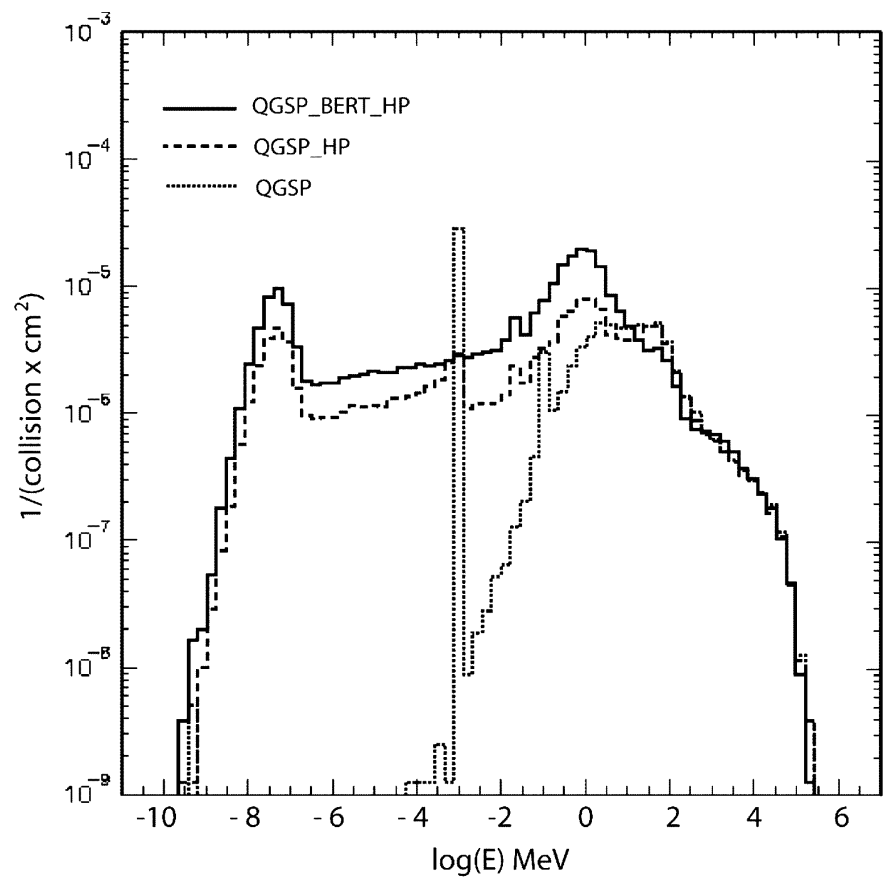

Fig. 16. Neutron energy spectrum on the $7830 \mathrm{~mm}$ plane, as obtained using QGSP_BERT_HP, QGSP_HP and QGSP.

This is due both to the HP extension for neutrons and to the Bertini cascade. In fact, the other physics lists show bands very similar to those obtained with QGSP_BERT_HP in the case of the charged particles distributions. For example, Fig. 15 shows the same band related to the electron fluence.

Another important issue associated with the use of QGSP_BERT_HP is the disappearance of the peak at $1 \mathrm{keV}$ recorded in all scoring planes using LHEP, QGSP and their HP extensions physics lists: as already mentioned the peak is due to an artefact of the evaporation code of the parameterized model and disappears in the physics list where the Bertini cascade replaces it.

This is clearly seen in Fig. 16 which is the neutron energy spectrum obtained with QGSP_BERT_HP, QGSP_HP and QGSP on the plane located at $7830 \mathrm{~mm}$ from the $\mathrm{LHCb}$ vertex.

The data reported in this article have been calculated on scoring planes properly positioned in order to avoid overlapping with the LHCb setup.

New features in Geant4 to avoid this overlapping issue are under preparation and testing. Furthermore more appropriate physics lists are envisaged.

Though beyond the scope of the present paper, further validation based on some similar experimental data is needed.

\section{CONCLUSION}

This paper describes the feasibility of performing background radiation studies, making use of the Geant 4 toolkit applied to $\mathrm{LHCb}$.

The prepared calculation module provides all the basic properties necessary to perform such studies. In particular, the scoring algorithm and the calculation of the quantities can be integrated in the built-in functions of Geant4. However, the choice of the scoring planes was influenced by the geometry 
constraint related to the impossibility to overlap the existing LHCb setup.

The physics lists considered in this study are all included in the normal Geant4 package. Only QGSP_BERT_HP needed some tuning to work properly.

The simulation results on the four scoring planes show the expected increase of the fluence (especially for neutrons) moving through the LHCb setup. The results of the comparison between similar physics lists covering the same energy range show that there are some disagreements, but the differences over the whole energy range are small. However, the complete explanation of these differences needs further investigations.

As expected, QGSP and LHEP are inadequate to studies involving low energy neutrons because of the limited energy range coverage.

The energy spectra obtained with QGSP_BERT_HP physics list are closest to those expected.

\section{ACKNOWLEDGMENT}

The authors would like to thank the Geant4 CERN group for helpful and fruitful discussions.

\section{REFERENCES}

[1] S. Amato, LHCb Tech. Proposal CERN-LHCC-98-004, 1998.
[2] R. Antunes Nobrega, LHCb reoptimized detector-Design and performance, CERN-LHCC-2003-030, 2003.

[3] K. Gill, "Radiation damage by neutrons and photons to silicon detectors," Nucl. Instr. Meth. A, vol. 322, pp. 177-188, 1992.

[4] M. Edwards, G. Hall, and S. Sotthibandhu, "Neutron radiation damage studies of silicon detectors," Nucl. Instrum. Methods Phys. Res. A, vol. A310, pp. 283-286, 1991.

[5] A. Vasilescu and G. Lindström, Notes on the Fluence Normalisation Based on the NIEL Scaling Hypothesis, ROSE/TN/2000-02, 2000.

[6] B. C. MacEvoy, Defect Evolution in Silicon Detector Material, ROSE/TN/96-1, 1996.

[7] A. Vasilescu, The NIEL Scaling Hypothesis Applied to Neutron Spectra of Irradiation Facilities and in the ATLAS and CMS SCT, ROSE/TN/97-2, 1997.

[8] S. Agostinelli, "Geant4-A simulation toolkit," Nucl. Instrum. Methods Phys. Res. A, vol. A506, pp. 250-303, 2003.

[9] J. Allison, "Geant4 developments and applications," IEEE Trans. Nucl. Sci., vol. 53, no. 1, pp. 270-278, Feb. 2006

[10] G. Corti, "Software for the LHCb experiment," IEEE Trans. Nucl. Sci., to be published.

[11] ASTM E722-93: Standard Practice for Characterizing Neutron Fluence Spectra in Terms of an Equivalent Monoenergetic Neutron Fluence for Radiation Hardness Testing of Electronics, American Society for Testing and Materials, Annual Book of ASTM Standards, 1993.

[12] J. P. Wellisch, "Hadronic physics simulation engines for Geant4," presented at the Proc. Monte Carlo 2005, Chattanooga, TN.

[13] J. P. Wellisch, "Hadronic shower simulation with Geant4," in Proc. CALOR '97, Tucson, AZ, 1997, pp. 522-529.

[14] T. Sjöstrand, P. Edén, C. Friberg, L. Lönnblad, G. Miu, S. Mrenna, and E. Norrbin, "High-Energy physics event generation with Pythia 6.1," Comp. Phys. Comm., vol. 135, pp. 238-259, 2001. 